\title{
Update on Colorectal Carcinomatosis-From Molecular Biology to Diagnosis and Management
}

\author{
Sandra F. Martins ${ }^{1,2,3}$, Adhemar Longatto Filho ${ }^{1,2,4 *}$ \\ ${ }^{1}$ Life and Health Sciences Research Institute (ICVS), School of Health Sciences, University of Minho, Braga, Portugal \\ ${ }^{2}$ ICVS/3B’s-PT Government Associate Laboratory, Braga/Guimarães, Portugal \\ ${ }^{3}$ Surgery Department, Hospital Center of Trás-os-Montes e Alto Douro, Vila Real, Portugal \\ ${ }^{4}$ Laboratory of Medical Investigation (LIM), Faculty of Medicine, University of São Paulo, São Paulo, Brazil \\ Email: sandramartins@ecsaude.uminho.pt, *longatto@ecsaude.uminho.pt
}

Received December 30, 2012; revised January 29, 2013; accepted February 7, 2013

Copyright (c) 2013 Sandra F. Martins, Adhemar Longatto Filho. This is an open access article distributed under the Creative Commons Attribution License, which permits unrestricted use, distribution, and reproduction in any medium, provided the original work is properly cited.

\begin{abstract}
After liver metastases, Peritoneal Carcinomatosis (PC) is the second most frequent cause of death in patients with Colorectal Cancer (CRC), although the precise incidence of Colorectal Cancer Peritoneal Carcinomatosis is not known, as the majority of the diagnostic studies cannot detect the disease in its initial stages, nowadays, the diagnosis of peritoneal carcinomatosis remains a challenge. The molecular biology of PC is only just beginning to be understood, future knowledge will permit not only identify novel strategies for PC prevention, but also contribute to therapeutic advances, through the development of molecular targeted therapies. The authors performed a literature revision about the Molecular Biology, Diagnosis and Management of Colorectal Cancer Peritoneal carcinomatosis.
\end{abstract}

Keywords: Peritoneal Carcinomatosis; Colorectal Cancer; Molecular Biology; Diagnosis; Management

\section{Introduction}

Peritoneal carcinomatosis (PC) consisted in the implantation of tumour cells throughout the peritoneal cavity [1] and, until recently, has been associated with a poor prognosis and considered a almost untreatable condition $[1,2]$.

In Colorectal Cancer (CRC), the precise incidence of PC is not known, as the majority of the CRC follow-up studies cannot detect the disease in its initial stages [2]. It is estimated that, at the time of diagnosis, the peritoneal surface is already involved in $10 \%-15 \%$ of cases [2], and that, after CRC curative surgical resection, PC occurs in up to $50 \%$ of patients [2,3]: $4 \%-19 \%$ during follow-up time and in almost $44 \%$ of the patients who require relaparotomy [4]. In 25\% of patients, CRC recurrence is confined to the peritoneal cavity $[2,4]$.

After liver metastases, $\mathrm{PC}$ is the second most frequent cause of death in patients with CRC $[1,4]$ and it is present in $40 \%-80 \%$ of patients who die from CRC [4].

Natural history studies show that Colorectal cancer PC is considered a terminal disease, with an median survival of 6 - 8 months [3,5], so treatment of this dissemi-

${ }^{*}$ Corresponding author. nated condition represents one of the Oncology limits [2,5]. Occasionally, patients needed a surgical intervenetion for intestinal occlusion, haemorrhage, or perforation caused by CP but most realized laparotomies for taking biopsies and then being referred to their oncologists for treatment with systemic chemotherapy [2]. Nowadays, with the introduction of locoregional therapies, promising results are suggested by several centers, namely cytoreductive surgery (CRS) combined with intraoperative intraperitoneal chemotherapy (PIC) without/with hyperthermia $[1,3]$.

\section{Molecular Biology of Colorectal Cancer Peritoneal Carcinomatosis}

Presently, our understanding of the molecular events involved in PC is ill-understood [6], future knowledge will permit not only identify novel strategies for PC prevention, but also contribute to therapeutic advances, through the development of molecular targeted therapies [7].

The development of PC involves a complex sequence of continuous and interdependent steps [8-10], known as the "peritoneal metastatic cascade" [6]; including cell 
shedding and transport, adhesion to the mesothelial layer, invasion and proliferation into the submesothelial stroma, and potential access to the systemic circulation $[9,10]$.

In the Peritoneal Tumour Dissemination step, the tumour cells are release from the primary tumour and gain access to the peritoneal cavity [6,7]. This can occur by several mechanisms namely, spontaneous exfoliation of tumour cells from the primary tumour, by iatrogenic or spontaneous perforation of the primary tumour or from transected lymphatics and blood vessels during tumour surgical resection [7]. After this, cancer cells are seeded in the peritoneal cavity and factors like gravity, peristaltic abdominal viscera movement and the flow of ascitic fluid resulting from the negative pressure exerted by diaphragm movements, contribute for the final destination of tumour cells, that adheres to the mesotelium, in the Mesothelial Adhesion step, and then can penetrate the mesothelial layer, gaining access to the submesothelial connective tissue, in the Mesothelial Invasion step, and then invading the underlying connective tissue; this environment provides the necessary scaffold for tumour proliferation that result in the establishment of metastatic tumour deposit, in the Stromal Invasion and Proliferation step. The final step involves the Induction of Angiogenesis to sustain tumour proliferation and enable further metastatic growth [6,7].

Some of the molecules involved in the "peritoneal metastatic cascade" have already been identified. In the Peritoneal Tumour Dissemination step, the detachment of cells from the primary tumour, can be mediated by the down-regulation of intercellular adhesion molecules on the tumor cell surface, E-cadherin, namely type I cadherins $[7,8,11]$. Next, free tumour cells invades the subperitoneal tissue, through matrix metalloproteinases (MMPs), such MMP-7, and directly attach to the submesothelial barrier connective tissue [through interaction with integrins, selectins and CD44, a lymphocyte homing molecules], in the Mesothelial Adhesion step [7,8,11]. In Mesothelial Invasion step, the production of cytokines, like interleukins, Endothelial Growth Factor (EGF), Hepatocyte Growth Factor (HGF), Vascular Endothelial Growth Factor-C (VEGF-C); induces the contraction of mesothelial cells exposing the submesothelial basement membrane and tumor cells can adhere to the submesothelial connective tissue through the interaction of integrins. The invasion of subperitoneal tissue requires the degradation of the peritoneal blood barrier by motility factors and matrix proteinases, the MMPs may play a central role Stromal Invasion and Proliferation step like urokinase plasminogen activating system. The next step, Induction of angiogenesis in the subperitoneal space is mediated by the production of VEGF-A and VEGF-C [7].

Another mechanism is described to peritoneal cancer dissemination, the translymphatic process; according to this process the peritoneal free cancer cells gain access to the subperitoneal lymphatic spaces through lymphatic stomata and milky spots that are abundant in the greater omentum, appendices epiploicae of the colon, inferior surface of the diaphragm, falciform ligament, Douglas pouch and small bowel mesentery. On the other hand the peritoneum covering the liver and the serosal surface of small bowel and spleen are devoid of lymphatic stomata as well as milky spots and thus are involved in peritoneal dissemination of cancer cells only in the late stage of peritoneal carcinomatosis [7].

\section{Diagnosis of Colorectal Cancer Peritoneal Carcinomatosis}

CRC is one of the most frequent cause of PC [12], but the diagnosis, of this condition, at initial stages is very difficult [2], as it can be limited to the ascitic fluid [13]. Future improvements in earlier diagnosis are essential to prevent unnecessary laparotomies and to select the patients in whom complete cytoreduction is feasible [14].

Computed tomography (CT) is generally considered as the first-choice modality for detecting PC [14], with varying sensitivity results ranging widely from $17 \%$ to 54\% [13] so, limited capacity for demonstrating PC [14]. Most CT scan findings are nonspecific; on the one hand, both neoplastic and non-neoplastic pathologies can present as soft-tissue peritoneal masses, with or without ascites [15]; on the other hand, TC sensibility depends on factors such as size, site, tumour deposit morphology, presence of ascites and paucity of intraabdominal fat [13]. Peritoneal tumor deposits are often small, and may be few in number or may extend along the parietal or visceral peritoneum and cause omental caking [13,15].

Some studies have demonstrated improved lesion detection with magnetic resonance imaging (MRI) [16,17], but not all [18].

Early detection of recurrence and high sensitivity and specificity has been reported with ${ }^{18}$ F-FDG PET [19]. Yoshiko Bamba et al. [14] document levels of detectability of PC from CRC using PET/CT of $82.6 \%$.

Evaluation of PC with ${ }^{18}$ F-FDG PET studies can identify two distinct patterns of glucose metabolism that appear to predict either nodular or diffuse peritoneal pathology and should alert the clinician to the possibility of PC [13]. It is most suitable in patients with high tumor markers and negative or uncertain conventional imaging data and in selecting patients for complete cytoreduction [20].

\section{Management of CRC Peritoneal Carcinomatosis}

The management of PC from CRC is evolving. In recent 
years, with the introduction of regional therapy and new chemotherapeutic and biologic agents, the outcomes for these patients has improved [21] but careful patient selection is needed, because of its significant but acceptable morbidity/mortality and cost $[10,22]$. Results of large multicentre studies have identified several prognostic factors that can be used to improve selection of CRC with carcinomatosis who will benefit from cytoreductive surgery (CRS) with hyperthermic intraperitoneal chemotherapy (HIPEC). The extent of colorectal carcinomatosis has been shown to be the most important prognostic factor determining survival [21].

Actually, the efficacy of CRS and HIPEC as a treatment option for PC from CRC is an established part of the oncologic literature $[21,23]$ and CRS in combination with HIPEC for peritoneal surface disease has been demonstrated to produce survival outcomes similar to liver resection for hepatic metastases [24].

The aim of surgery is to remove all macroscopically visible tumor implants from the visceral and parietal peritoneum; thus, cytoreductive surgery consisted of a variety of peritonectomy procedures, whose extent depends of peritoneum tumor infiltration, but often multivisceral resections are necessary to achieve complete macrosopical cytoreduction [25,26].

After complete cytoreduction, HIPEC is realized and the abdominal and pelvic spaces are flooded by a warm chemotherapy solution, the heated circulation was maintained for one additional hour. During the time of perfusion, the operating table is moved in all possible directions to guarantee optimal distribution of the heated agent within the abdomen [23]. These treatments may be continued with early postoperative intraperitoneal chemotherapy (EPIC), using cell cycle-specific drugs that should contact all visceral and parietal surfaces because their use precedes the development of abdominal adhesions [23].

Until more data become available, the management strategy supported by the literature is CRS plus HIPEC. Based on current evidence, modern chemotherapies for patients with CRC carcinomatosis achieves a median survival of 24 months compared to the 63 months achived for CRS plus HIPEC, with a 5-year survival of 51\% [21, 23].

\section{Conclusions}

Peritoneal Carcinomatosis from Colorectal cancer is probably an underdiagnosed condition, as earlier diagnosis of this condition is very difficult.

Recent advances in the molecular biology knowledge will not only enable only identify novel strategies for PC prevention and earlier detection, but also improve treatment through the development of molecular targeted therapies.

\section{REFERENCES}

[1] V. E. Lemmens, I. H. de Hingh, et al., "Predictors and Survival of Synchronous Peritoneal Carcinomatosis of Colorectal Origin: A Population-Based Study,” International Journal of Cancer, Vol. 128, No. 11, 2011, pp. $2717-$ 2725. doi:10.1002/ijc.25596

[2] A. Gómez Portilla, I. Cendoya, I. López de Tejada, I. Olabarría, C. M. de Lecea, L. Magrach, A. Gil, J. Echevarría, M. Valdovinos and I. Larrabide, "Peritoneal Carcinomatosis of Colorectal Origin. Current Treatment. Review and Update,” Revista Española de Enfermedades Digestivas, Vol. 97, No. 10, 2005. doi:10.4321/S1130-01082005001000005

[3] D. Elias, F. Gilly, F. Boutitie, F. Quenet, J.-M. Bereder, B. Mansvelt, G. Lorimier, P. Dubè and O. Glehen, "Peritoneal Colorectal Carcinomatosis Treated with Surgery and Perioperative Intraperitoneal Chemotherapy: Retrospective Analysis of 523 Patients from a Multicentric French Study," Journal of Clinical Oncology, Vol. 28, No. 1, 2010, pp. 63-68.

[4] A. Macrì, E. Saladino, V. Bartolo, V. Adamo, G. Altavilla E. Mondello, G. Condemi, A. Sinardi and C. Famulari, "Peritoneal Carcinomatosis of Colorectal Origin," World Journal of Gastrointestinal Oncology, Vol. 2, No. 2, 2010, pp. 98-101. doi:10.4251/wjgo.v2.i2.98

[5] D. Goéré, "Incidence and Prognosis of Synchronous Colorectal Carcinomatosis: Evolution since 1985?” Future Oncology, Vol. 7, No. 11, 2011, pp. 1265-1268.

[6] D. Jayne, "Molecular Biology of Peritoneal Carcinomatosis. Peritoneal Carcinomatosis: A Multidisciplinary Approach,” Springer, Berlin, 2007, pp. 21-33.

[7] S. Kusamura, D. Baratti, N. Zaffaroni, R. Villa, B. Laterza, M. R. Balestra, et al., "Pathophysiology and Biology of Peritoneal Carcinomatosis," World Journal of Gastrointestinal Oncology, Vol. 2, No. 1, 2010, pp. 1218.

[8] D. G. Jayne, "The Molecular Biology of Peritoneal Carcinomatosis from Gastrointestinal Cancer," Annals Academy of Medicine Singapore, Vol. 32, No. 2, 2003, pp. 219-225.

[9] P. Wim and M. E. B. Ceelen, "Peritoneal Minimal Residual Disease in Colorectal Cancer: Mechanisms, Prevention, and Treatment," The Lancet Oncology, Vol. 10, No. 1, 2009, pp. 72-79. doi:10.1016/S1470-2045(08)70335-8

[10] E. M. V. de Cuba, R. Kwakman, M. van Egmond, L. J. W. Bosch, H. J. Bonjer, G. A. Meijer and E. A. te Velde, "Understanding Molecular Mechanisms in Peritoneal Dissemination of Colorectal Cancer: Future Possibilities for Personalised Treatment by Use of Biomarkers,” Virchows Archiv, Vol. 461, No. 3, 2012, pp. 231-243. doi:10.1007/s00428-012-1287-y

[11] S. Zerhouni and J. A. Mccart, "Peritoneal Carcinomatosis of Colorectal Origin. Is There a Role for Cytoreductive Surgery and Hyperthermic Intraperitoneal Chemotherapy?” Colorectal Disease, Vol. 11, No. 2, 2012, pp. 1419.

[12] A. J. Suleiman, N. A. Uraiqat and O. A. Al-Imam, "Peritoneal Carcinomatosis Computerized Tomography Scans 
Findings and Causes,” JRMS, Vol. 11, No. 1, 2004, pp. 63-66.

[13] A. Turlakow, H. W. Yeung, A. S. Salmon, H. A. Macapinlac and S. M. Larson, "Peritoneal Carcinomatosis: Role of (18)F-FDG PET,” Journal of Nuclear Medicine: Official Publication, Society of Nuclear Medicine, Vol. 44, No. 9, 2003, pp. 1407-1112.

[14] Y. Bamba, M. Itabashi and S. Kameoka, “Clinical Use of $\mathrm{PET} / \mathrm{CT}$ in Peritoneal Carcinomatosis from Colorectal Cancer," Hepatogastroenterology, Vol. 59, No. 117, 2012, pp. 1408-1411.

[15] S. Smiti and K. V. Rajagopal, "CT Mimics of Peritoneal Carcinomatosis,” Abdominal and Gastrointestinal Radiology, Vol. 20, No. 1, 2010, pp. 58-62.

[16] R. N. Low, R. M. Barone, C. Lacey, J. S. Sigeti, G. D. Alzate and C. P. Sebrechts, "Peritoneal Tumor: MR Imaging with Dilute Oral Barium and Intravenous Gadolinium-Containing Contrast Agents Compared with Unenhanced MR Imaging and CT,” Radiology, Vol. 204, No. 2, 1997, pp. 513-520.

[17] U. Tateishi, T. Hasegawa, M. Kusumoto, T. Oyama, H. Ishikawa and N. Moriyama, "Desmoplastic Small Round Cell Tumor: Imaging Findings Associated with Clinicopathologic Features," Journal of Computer Assisted Tomography, Vol. 26, No. 4, 2002, pp. 579-583. doi:10.1097/00004728-200207000-00018

[18] M. G. Brizi, L. Natale, R. Manfredi, B. Barbaro, A. Vecchiolo and P. Marano, "Staging of Pancreatic Ductal Adenocarcinoma with Spiral CT and MRI,” Rays, Vol. 26, No. 2, 2001, pp. 151-159.

[19] J. Esquivel, T. C. Chua, A. Stojadinovic, et al., "Accuracy and Clinical Relevance of Computed Tomography Scan Interpretation of Peritoneal Cancer Index in Colorectal Cancer Peritoneal Carcinomatosis: A Multi-Institutional Study,” Journal of Surgical Oncology, Vol. 102, No. 6, 2010, pp. 565-570. doi:10.1002/jso.21601
[20] A. M. De Gaetano, M. L. Calcagni, V. Rufini, V. Valenza, A. Giordano and L. Bonomo, "Imaging of Peritoneal Carcinomatosis with FDG PET-CT: Diagnostic Patterns, Case Examples and Pitfalls," Abdominal Imaging, Vol. 34, No. 3, 2009, pp. 391-402. doi:10.1007/s00261-008-9405-7

[21] F. Mohamed, T. Cecil, B. Moran and P. Sugarbaker, “A New Standard of Care for the Management of Peritoneal Surface Malignancy,” Current Oncology, Vol. 18, No. 2, 2011, pp. 84-96. doi:10.3747/co.v18i2.663

[22] E. Cotte, G. Passot, F. Mohamed, D. Vaudoyer, F. N. Gilly and O. Glehen, "Management of Peritoneal Carcinomatosis from Colorectal Cancer: Current State of Practice,” The Cancer Journal, Vol. 15, No. 3, 2009, pp. 243248. doi:10.1097/PPO.0b013e3181a58d67

[23] P. H. Sugarbaker, “Cytoreductive Surgery plus Hyperthermic Perioperative Chemotherapy for Selected Patients with Peritoneal Metastases from Colorectal Cancer: A New Standard of Care or an Experimental Approach?” The Lancet Oncology, Vol. 13, No. 8, 2012, pp. e362e369.

[24] C. U. Ihemelandu, P. Shen, J. H. Stewart, K. Votanopoulos and E. A. Levine, "Management of Peritoneal CarciNomatosis from Colorectal Cancer," Seminars in Oncology, Vol. 38, No. 4, 2011, pp. 568-575. doi:10.1053/j.seminoncol.2011.05.011

[25] P. Piso, M. H. Dahlke, N. Ghali, I. Iesalnieks, M. Loss, F. Popp, et al., "Multimodality Treatment of Per Itoneal Carcinomatosis from Colorectal Cancer: First Results of a New German Centre for Peritoneal Surface Malignancies,” International Journal of Colorectal Disease, Vol. 22, No. 11, 2007, pp. 1295-1300. doi:10.1007/s00384-007-0313-z

[26] P. H. Sugarbaker, "Management of Peritoneal-Surface Malignancy: The Surgeon's Role,” Langenbeck's Archives of Surgery, Vol. 384, No. 6, 1999, pp. 576-587. doi:10.1007/s004230050246 